\title{
Does the 5-strand-graft have superior biomechanical behaviour than 4-strand-graft during $A C L$ reconstruction?
}

\author{
D. Pavan 1, G. Pitarresi ${ }^{2}$, F. Morello1, F. Monachino 1 , A. Sanfilippo 1, \\ A. D'Arienzo ${ }^{3}$, L. Camarda ${ }^{1}$ \\ 1 Department of Orthopaedic Surgery, University of Palermo (Di.Chir.On.S), Palermo, Italy \\ 2 Department of Industrial and Digital Innovation (DIID) Chemical, Management, Computer, Mechanical \\ Engineering, Viale delle Scienze ed.8, I-90128 Palermo \\ 3 Department of Orthopaedic Surgery, University of Pisa, Pisa, Italy
}

\section{CORRESPONDING AUTHOR: \\ Lawrence Camarda, \\ Department of Orthopaedic Surgery \\ (DiChirOnS) \\ University of Palermo \\ Via del Vespro, Palermo, Italy \\ E-mail: lawrence.camarda@unipa.it}

DOI:

10.32098/mltj.03.2019.12

LEVEL OF EVIDENCE:

Controlled Laboratory Study

\begin{abstract}
SUMMARY
Purpose. This study aimed to compare the biomechanical proprieties of 4 and 5 -strandgrafts.

Methods. For the present study, fresh-frozen bovine common digital extensor tendons were used. Tendon grafts were prepared and sized to have a length of almost $27 \mathrm{~cm}$ and a cylinder 4 strand diameter of $8 \mathrm{~mm}$. In half of all samples (Group $A, n=12$,), the graft was formed by 2 bundles duplicated around a metallic rod creating a 4-strand-tendon construct. In the other half of samples (Group B, n= 12,) 5 -strand-tendon construct was created duplicating a single tendon around the rod, and tripling the other one. The grafts were preconditioned at $50 \mathrm{~N}$ for $10 \mathrm{~min}$, followed by 1,000 cyclic loading between 50 and $250 \mathrm{~N}$. Load-to-failure test was then carried out at a rate of $1 \mathrm{~mm} / \mathrm{s}$.

Results. No statistically significant differences were found between two groups concerning cyclic elongation at the $500^{\text {th }}$ cycle and at the final cycle. An increased stiffness was observed in the Group B during cyclic loads and at pull-out $(\mathrm{p}<0.05)$. Significant differences were noted at the ultimate load-to-failure between Group A $(1533 \pm 454 \mathrm{~N})$ and Group B $(1139 \pm 276$ N) $(\mathrm{p}<0.05)$.

Conclusion. This study showed that both graft construct appears to be biomechanically effective in a bovine tendon model. 5-strand-graft showed an increased stiffness and a decreased ultimate load-to-failure comparing to the 4-strand-graft construct. Biomechanically, no real benefit could be observed in the clinical setting increasing the numbers of strands used for the ACL reconstructive surgery.
\end{abstract}

\section{KEY WORDS}

5-strand-graft; 4-strand-graft; graft diameter; ACL reconstruction; graft; knee biomechanics.

\section{INTRODUCTION}

Recently, an increase of knowledge on the effect of the graft diameter on the success of ACL reconstruction surgery was observed. In fact, it was reported that a graft diameter $<8$ $\mathrm{mm}$ represents a high predictor for graft failure and ACL revision surgery (1). To reach the minimum of $8 \mathrm{~mm}$ graft diameter overall, the semitendinosus tendon is typically folded to create a 4-stranded-graft, resulting in a stiffer biomechanical construct compared to other autogenous tendon graft constructs (2). However, sometimes semitendinosus tendons harvest result in short tendons, making it impossible to quadruple the graft. In this circumstance, it could be a reliable option to harvest the gracilis tendon preparing a 5 -strand hamstring graft with a double-stranded gracilis and a tripled semintendinosus tendons (3, 4). Even if this surgical technique of graft preparing could help surgeons to increase graft diameter, tripling the tendon has been reported to present a biomechanical disadvantage, because 
of third limb elongation during loads, and in particular with evolution of fatigue cycling loads, suggesting a poorer long term performance of the triple-stranded tendon. This could compromise the tensile loading strength, predisposing to graft stress-shielded and limiting the physiological ligamentization process (5). However, creating the 5 -strand hamstring graft, each single graft limbs could share loads differently compared to 4-strands or tripled graft, better resisting to initial loads observed during the postoperative rehabilitation period. On the contrary, poor data are available at the moment regarding biomechanical behaviour of the 5-strand hamstring graft technique. Further, no study has compared the biomechanical proprieties of 4-strands and 5 -strands, created using double-stranded and tripled tendons.

The aim of the present study was to compare the biomechanical proprieties of 4 and 5-strand-grafts used during ACL reconstruction. The authors hypothesized that increasing the number of strands, as performed following the 5-strand-grafts technique, could positively influence the graft behaviour, reducing graft elongation during cyclic loads and increasing the graft stiffness.

\section{MATERIALS AND METHODS}

For the present study, 12 fresh-frozen bovine common digital extensor tendons were harvested from mature bovine ageing from 18 to 24 months. Each tendon was divided in half to get 24 samples. Tendon grafts were then prepared and sized to have a length of almost $27 \mathrm{~cm}$ and a cylinder 4 strand diameter of $8 \mathrm{~mm}$. All tendon grafts were immediately wrapped in a normal saline soaked gauze, stored at -38 ${ }^{\circ} \mathrm{C}$ and then thawed at room temperature $12 \mathrm{~h}$ before use. Continuous saline graft irrigation was performed throughout the preparation and mechanical testing to prevent drying (6). As per standard intra-operative technique, each tendon ends were separately whipstitched with no. 2 non-absorbable sutures (Ticron, Tyco, Waltham, MA) for a length of 30 $\mathrm{mm}$. All tendons were sutured by an expert knee surgeon. For the tests, an electro-mechanic two-columns universal testing machine (Instron 3367), equipped with a $30 \mathrm{KN}$ load cell (Instron Systems, Norwood, Massachusetts), was used. Since the experiments carried out require the measurement of load levels comprised in the range $50-1500 \mathrm{~N}$, the accuracy of the load cell was verified to be within $\pm 0.5 \%$ of the reading, which is considered sufficient for the evaluations made in this work. During the tests, each bovine tendon graft was looped around a cylindrical steel rood with $5 \mathrm{~mm}$ diameter, directly connected to the load cell through a clevis-like adapter $(7,8)$. On the opposite end, the samples were fixed by a Zwick Roell wedge-screw grip, clamping the samples for a length of $4 \mathrm{~cm}$. Graft fixation was set so that the distance from the clamp to the rod was $70 \mathrm{~mm}$, to simulate the intra-articular space of the ACL $(30 \mathrm{~mm})$ and femoral tunnel length $(40 \mathrm{~mm})$ that could be obtained with more recent femoral fixation devices (Figure 1).

During tripling, care was taken to pass all stitches through each graft strand, securing the free tendon limb to other strands. All sutures were applied using the same non-absorbable suture (No.2 Ticron, Tyco, Waltham, MA). The suture was passed when the graft was under a slight tensile load, to avoid permanent graft elongation, due to the slippage of the suture over the tendon tissue during loads $(9,10)$. Graft diameter of 4 and 5 -strand-tendon constructs was measured before tests using a graft diameter measurement guide with increments of $0.5 \mathrm{~mm}$ (Smith \& Nephew, Memphis, TN).

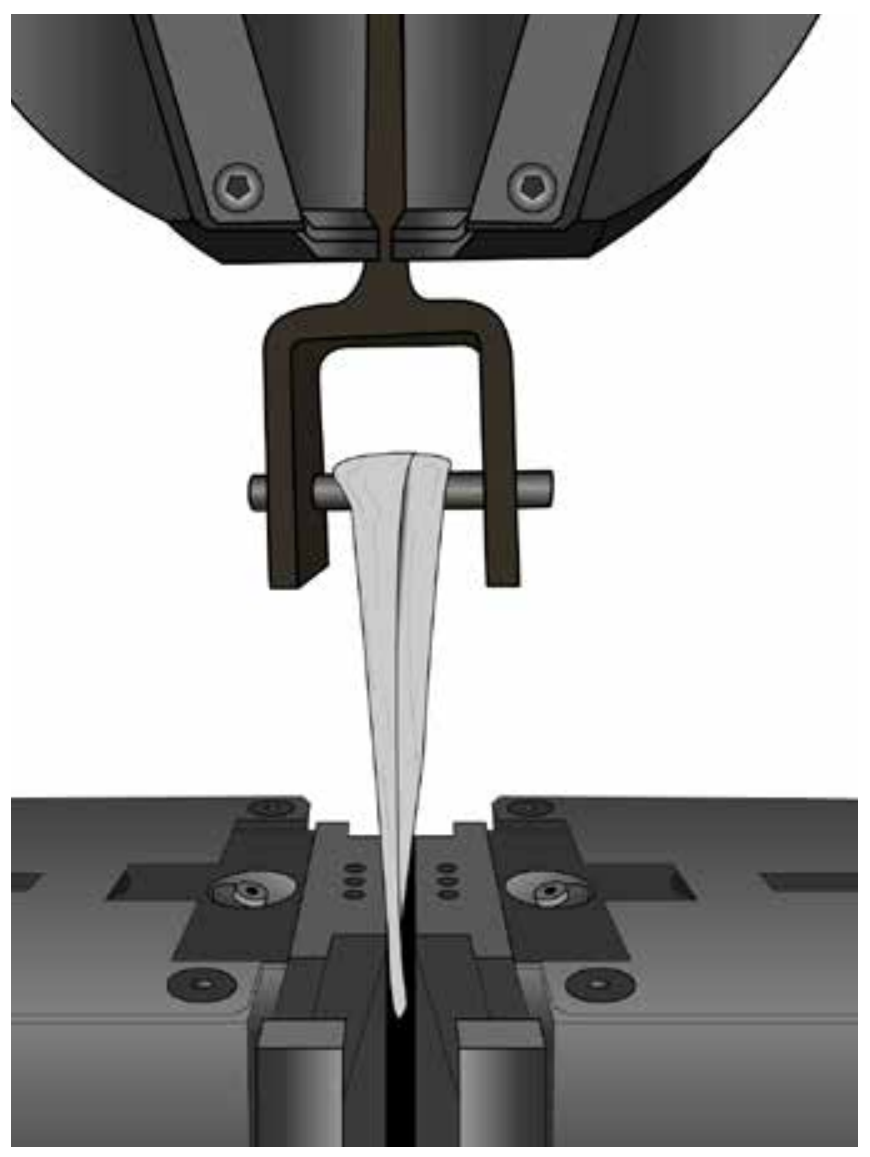

Figure 1. Illustration showing the experimental setup. In half of all samples (Group $A, n=12$ ), the graft was formed by 2 bundles duplicated around the rod creating a 4-strand-tendon construct. In the other half of samples (Group B, $n=12$,) 5 -strand-tendon construct was created duplicating a single tendon around the rod, and tripling the other one as shown in Figure 2a and Figure $2 \mathrm{~b}$. 


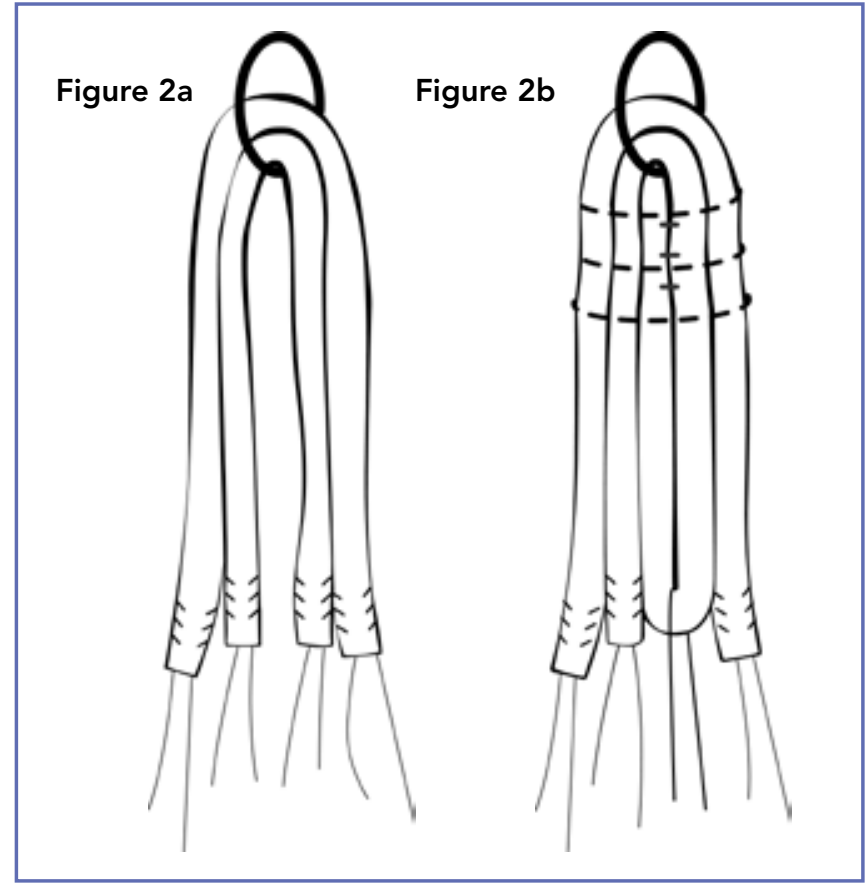

Figure 2. Illustration that show the two graft constructs used for the study. a) 4-strand-tendon b) the 5-strand-tendon

\section{Biomechanical testing}

A specific loading protocol was created, by exploiting the Instron Blue Hill v 2.0 remote control software of the testing machine. In particular, the Test Profiler feature has been used, allowing to set a sequence of loading patterns that can comprise triangle waves, ramps and holds in both load or displacement control. The load protocol set in this study comprised three successive stages:

1. static pre-conditioning by holding the tendon at a stable tensile load of $50 \mathrm{~N}$ for $10 \mathrm{~min}$ (kept constant by operating the machine in loading control, and corresponding to $\mathrm{T}_{\mathrm{a}-\mathrm{b}}$ in Figure 3);

2. 1,000 cyclic loading between 50 and $250 \mathrm{~N}$, with a triangle wave at $1 \mathrm{~Hz}$ (under load control, corresponding to $\mathrm{T}_{\text {b-j }}$ in Fig. 3);

3. final monotonic tensile loading up to failure performed in displacement control at a machine crosshead speed of 1 $\mathrm{mm} / \mathrm{s}$ (corresponding to $T_{j-i-\mathrm{r}}$ in Figure 3 ).

The preconditioning stage was performed in order to stabilize the graft's mechanical properties $(8,11,12)$. Cyclic loading between 50 and $250 \mathrm{~N}$ has been described to well simulate previously measured forces in the ACL during passive flexion-extension of the knee (12), while a frequency of 1 $\mathrm{Hz}$ simulates the reported frequency of walking (11). The number of 1,000 cycles was chosen to simulate an aggressive postoperative rehabilitation protocol of the knee ${ }^{7}$. The final monotonic stage, immediately following the cyclic testing, allowed the evaluation of the residual static stiffness and strength through the evaluation of the ultimate load at failure. The amount of graft elongation in response to cyclic loading and at failure were obtained from the testing machine crosshead displacement.

For each specimen, load-displacement curves were recorded and analysed to determine specific parameters. Specifically:

- the amplitude of graft elongation during a peak-to-peak fatigue cycle was determined, given by the difference in crosshead displacement between the load peak and the valley. Such amplitude was in particular measured at the first applied fatigue cycle $\left[\mathrm{L}_{1}\right.$ or $\mathrm{D}_{c-\mathrm{d}}$ in Figure $\left.3 \mathbf{b}\right]$, at the $500^{\text {th }}$ cycle $\left[\mathrm{L}_{500}\right.$ or $\mathrm{D}_{\text {e-f }}$ in Figure $\left.3 \mathbf{b}\right]$ and at the last cycle $\left[\mathrm{L}_{1000}\right.$ or $\mathrm{D}_{\mathrm{g}-\mathrm{h}}$ in Figure $\left.3 \mathbf{b}\right]$;

- the graft slippage $\left[\mathrm{L}_{4}\right.$ or $\mathrm{D}_{\text {ch }}$ in Figure $3 \mathbf{b}$ ] (the difference of graft accumulated elongation between the last and the first cycle (measured at the valleys of the cyclic loading);

- the cyclic elongation $0-500^{\text {th }}\left[\mathrm{D}_{0-500}\right.$ or $\mathrm{D}_{\mathrm{b}-\mathrm{f}}$ in Figure 3b], defined as the difference in crosshead displacement between the condition at the end of the $50-\mathrm{N}$ static pre-conditioning hold and the condition at the max applied load in the 500th fatigue cycle;

- the final elongation $\left[\mathrm{D}_{0-1000}\right.$ or $\mathrm{D}_{\mathrm{b}-\mathrm{h}}$ in Figure $3 \mathbf{b}$ ] (as previously but calculated at 1000th cycle);

- the initial stiffness $\left[\mathrm{K}_{10}\right.$ or $\mathrm{F}_{\mathrm{c}-\mathrm{d}} / \mathrm{D}_{\mathrm{c}-\mathrm{d}}$, i.e. the slope of the secant line joining minimum and maximum points of the loading wave in the load-displacement curve, measured at the 10th cycle);

- cyclic stiffness at 500th cycle $\left[\mathrm{K}_{500}\right.$ or $\left.\mathrm{F}_{\mathrm{e} . \mathrm{f}} / \mathrm{D}_{\mathrm{e} . \mathrm{f}}\right]$ (as previous but at the 500th cycle);

- pull-out stiffness $\left[\mathrm{K}_{\mathrm{L}}\right.$ or $\mathrm{F}_{\mathrm{i}-\mathrm{i}} / \mathrm{D}_{\mathrm{i}-\mathrm{i}}$, i.e. the initial slope of the load-displacement curve at the final monotonic loading. The initial slope corresponds to the steepest straight-line tangent to the curve;

- ultimate failure load [Fr, Figure 3b], i.e. the peak force of the final load-elongation curve.

The mechanism of final static failure for each specimen was also observed and recorded. All research was conducted ethically according to the international standards (13).

\section{Statistical analysis}

Data were analysed using SPSS statistical software, version 18.0 (SPSS, Inc., Chicago, IL, USA). Means and standard deviations were calculated for all parameters of each group. Grafts tensile properties between 4-strands and 5-strands graft groups were analysed using a paired Student's t-test 

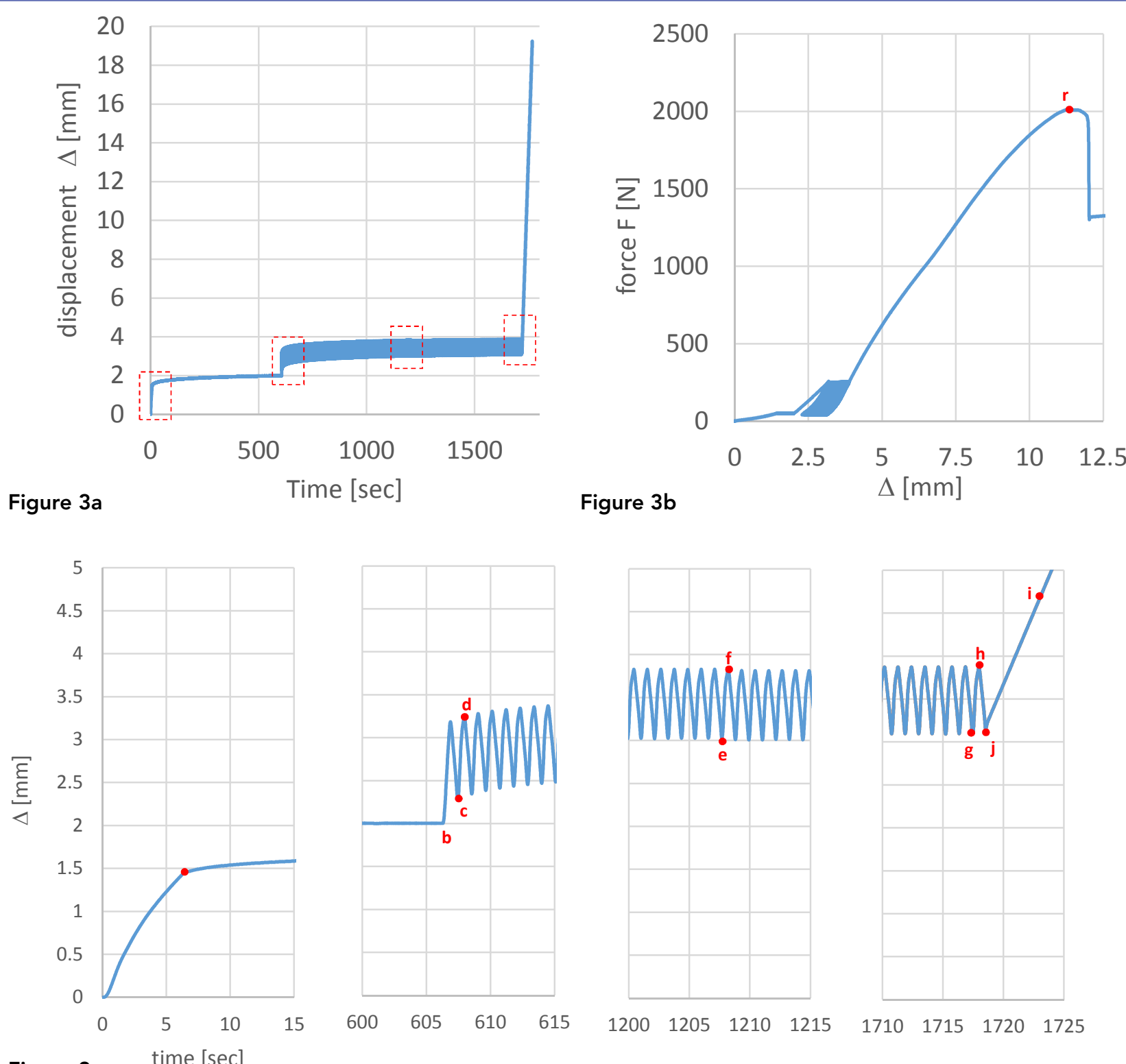

Figure 3c

Figure 3. typical experimental data: a) plot of machine crosshead displacement $\Delta$ versus time; b) plot of force $F$ versus $\Delta$; c) zoomed areas from Figure 3a, showing the definition of points used to evaluate the analysis parameters.

with a level of significance at $\alpha=0.05$ and a post hoc power of $\beta=0.9395$.

\section{RESULTS}

The quantitative parameters defined in the previous section are shown in Table I. Graft diameter of 4-strand-tendon constructs was $8 \mathrm{~mm}$ while in 4 -strand-tendon constructs was $10 \mathrm{~mm}$. All specimens were successfully tested and characterized. In all specimens of Group B (5 strands), failure occurred following partial breaking and then slipping of the tendons from the bottom serrated clamp. In 4 cases of Group A (4 strands) failure occurred as described in Group 1. In 8 cases failure occurred at the level of the cylindrical rod, while in 1 case failure occurred in the middle of the bundles. 
Table I. Mechanical properties of 4 and 5-strand grafts at cyclic loads and ultimate failure load. Data presented as mean \pm standard deviation. * $\mathrm{p}<0.05$ (statistically significative difference)

\begin{tabular}{|c|c|c|c|}
\hline & $\begin{array}{l}\text { Group A } \\
\text { (4-strands) }\end{array}$ & $\begin{array}{l}\text { Group B } \\
\text { (5-strands) }\end{array}$ & p-value \\
\hline Ultimate failure load [N] $(\mathrm{Fr})$ & $1533 \pm 454 *$ & $1140 \pm 276$ & 0.0193 \\
\hline Initial stiffness $[\mathrm{N} / \mathrm{mm}]\left(\mathrm{K}_{10}\right)$ & $225 \pm 48$ & $296 \pm 70 *$ & 0.0088 \\
\hline Cyclic stiffness at 500 th cycle $[\mathrm{N} / \mathrm{mm}]\left(\mathrm{K}_{500}\right)$ & $247 \pm 54$ & $331 \pm 69 *$ & 0.0069 \\
\hline Final stiffness at 1000 cycle $[\mathrm{N} / \mathrm{mm}]\left(\mathrm{K}_{\mathrm{L}}\right)$ & $273 \pm 56$ & $353 \pm 82 *$ & 0.0099 \\
\hline Elongation 0-500 $[\mathrm{mm}]\left(\mathrm{D}_{0-500}\right)$ & $1,6 \pm 0.2$ & $1,5 \pm 0.3$ & 0.2904 \\
\hline Slippage $[\mathrm{mm}](\mathrm{L} 4)$ & $0.9 \pm 0.3$ & $1.1 \pm 0.2$ & 0.1706 \\
\hline Graft elongation amplitude at firs cycle $[\mathrm{mm}]\left(\mathrm{L}_{1}\right)$ & $1 \pm 0.2$ & $0.8 \pm 0.2 *$ & 0.0102 \\
\hline Graft elongation amplitude 500th cycle $[\mathrm{mm}]\left(\mathrm{L}_{500}\right)$ & $0.9 \pm 0.2$ & $0.7 \pm 0.2 *$ & 0.0039 \\
\hline Graft elongation amplitude 1000th cycle $[\mathrm{mm}]\left(\mathrm{L}_{1000}\right)$ & $0.8 \pm 0.1$ & $0.6 \pm 0.1 *$ & 0.0054 \\
\hline
\end{tabular}

No statistically significant differences were found between two groups concerning slippage [L4], cyclic elongation at the $500^{\text {th }}$ cycle $\left[\mathrm{D}_{0-500}\right]$ and at the final cycle $\left[\mathrm{D}_{0-1000}\right]$. Statistically significant differences were detected for graft elongation amplitude in all measurements between the 2 groups [L1, L2 and L3] (Table I). The mean initial stiffness $\left[\mathrm{K}_{10}\right]$ was $225 \pm 48 \mathrm{~N} / \mathrm{mm}$ in group $A$ and $296 \pm 70 \mathrm{~N} / \mathrm{mm}$ in group $\mathrm{B}$. The mean cyclic stiffness $\left[\mathrm{K}_{500}\right]$ was respectively $247 \pm 54 \mathrm{~N} / \mathrm{mm}$ and $331 \pm 79 \mathrm{~N} / \mathrm{mm}$ in group $A$ and B while stiffness at pull-out $\left[\mathrm{K}_{\mathrm{L}}\right]$ was $272 \pm 56 \mathrm{~N} / \mathrm{mm} 353$ $\pm 82 \mathrm{~N} / \mathrm{mm}$. All differences were statistically significant $(\mathrm{p}<0,05)$. Concerning the ultimate load-to-failure, significant differences were noted between Group A (1533 \pm 454 N) and Group B $(1139 \pm 276$ N) $(\mathrm{p}<0.05)$.

\section{DISCUSSION}

The most important finding of the present study was that in a bovine model the 5 -strand-graft presented a biomechanical behaviour comparable to the well-known 4-strand-graft. Specifically, no differences were observed between two constructs in terms of graft elongation during cyclic loads. However, the 5 -strand-graft presented an increased stiffness during cyclic loads and a reduced ultimate failure load.

In the recent years, advance in preoperative evaluation was observed to predict semitendinosus and gracilis graft diameter to identify patients at risk of undersized grafts during ACL reconstructive surgery $(14,15)$. In fact, small-diameter grafts have been demonstrated to have a weaker ultimate failure load (16), and are associated to an increased revision rate of ACL reconstructive surgery (1). For this reason, some authors have suggested to quadruple the semitendinous graft in order to reduce the donor site morbidity of gracilis harvesting and to obtain a larger graft, minimizing the risk of future graft failure (17). In this circumstance, femoral and tibial cortical suspensory buttons should be used for the final graft fixation resulting in an increase of technical difficulty of the reconstructive surgery (all-inside technique). Indeed, some authors have described different methods for folding the semitendinous and gracilis, in order to create a 5-strand-hamstring graft with enough length to allow a tibial fixation using an interference screw. Despite the less technical difficulty of the use of 5-strand-graft, doubts are present concerning the biomechanical properties of the tripled semitendinousus graft used to create the 5 -strand-graft. In a study on tripled tendons, Snow et al observed that there was no mechanical difference in the overall properties between a doubled tendon and a tripled tendon graft when used in association with suspensory fixation. However, a cyclic elongation with a decreased tensile stress was observed in the third limb of the tripled tendon in comparison with the doubled portion. In addition, no study is present concerning the biomechanical behaviour of a 5-strand graft preparation using a double tendon and a tripled tendon. In the present study, the graft was tripled similarly to the technique described by Lavery et al (3), suturing the third limb to the other 4 strands. Specifically, the third limb was sutured to the other two strands of the same graft, and all three strands were sutured to the other 2 of the remnant tendon graft. Following this configuration, a greater diameter was obtained and an increased stiffness was recorded during all phases of cyclic loads. Furthermore, the graft elongation amplitude in all measurements was reduced in the 5-strand-graft group, justifying an increased stiffness of the construct during loads. These results could suggest that the third strand could contribute to the tension of 
the 5-strands-graft. Another explanation could be that the whip-stitched portion of the 5-strands-graft might exhibit a different intrinsic stiffness rather than a free quadruple bundle. In fact, if a part of the graft is stitched, and the stitching is able to stiffen the graft locally, the overall stiffness of the graft will then be higher (6). This finding significantly differs from what have been reported recently in literature $(18,19)$, and could depend by many factors such us stripling technique, suture used for graft preparation and biomechanical protocol of graft testing. In a recent study of 5 -strand-graft with a free strand of semitendinosus (i.e. not tripling the graft) (18), Vaillant et al found that a fifth limb added to a four-strand hamstring did not significantly change stiffness or displacement (18). In this study, a no. 1 Vicryl suture was used and tied around the entire graft to tubularize the construct (18). Recenlty, Broadhead et al comparing 4-strand and 5-strand constructs did not show differences between two constructs concerning stress relaxation, ultimate failure load and stiffness (19). However, their protocol included only 50 cyclic loads between 10 to $100 \mathrm{~N}$. Further, the third limb was secured only suturing directly the tendon end of the third limb to the endobutton following 5 square knots (19). In the present study the tripled tendon was created suturing the free tendon limb to others strands of the same tendon. Further, the 5 -strand was created suturing together all strands of the femoral portion of the graft, aiming to increase the contribution of the third limb during loads. In addition, 1,000 cyclic loads were applied to the graft to simulate an aggressive postoperative rehabilitation protocol of the knee (7), and to potentially detect any biomechanical differences between grafts. However, it was found that the graft elongation during cyclic loads was similar for both graft constructs tested. This could be explained because the majority of graft elongation could be generated at the graft-rod location, as both constructs have two strands looped around the rod. In addition, the similar elongation between the two graft configurations could be due to the reduced contribution of the third limb in the tripled graft. In fact, this limb can be stretched only by taking some load from the neighbour limb to which it is stitched. Even so, the amount of stress in the third limb is likely to be smaller than other limbs coming from strands folded across the rod. On the basis of the results of the study, it is reasonable to assume that no real benefit could be observed in the clinical setting increasing the numbers of strands used for the reconstructive surgery. In fact, no differences could be observed in the immediate postoperative time. However, the increased graft diameter with a greater graft-bone contact potentially could increase the graft incorporation, thereby reducing recovery time and the risk of re-rupture (20). Even these theoretical assumptions are valid, further studies are required to eval- uated the risk of rerupture after ACL reconstruction with 5 -strands.

The present study has some limitations. At first, bovine tendons were used in place of human hamstrings. However, their immediate availability and low-cost represent the major advantages of their use. Further, bovine common digital extensor tendons present viscoelastic and structural properties comparable to a graft composed of a double loop of semitendinosus and gracilis tendons from humans (21, 22). Dividing the bovine tendons in half, we also tried to limit the differences in the interaction between the tendon and sutures when compared to human grafts. Second, this is an in-vitro controlled biomechanical study and some variables were not investigated such as the interaction between the tendon and several methods of graft fixation. This aspect can affect the results in vivo. Also, the use of other femoral fixation systems could show different results even if the use of a cylindrical metal rod partially reproduces common cortical fixation systems.

\section{CONCLUSION}

This study showed that both graft construct appears to be biomechanically effective in a bovine tendon model. However, 5-strand-graft showed an increased stiffness and a decreased ultimate load-to-failure comparing to the 4 -strand-graft construct. This information should prove to be helpful for surgeons in choosing the hamstring graft preparation technique to increase graft diameter in case of undersized hamstring autograft.

\section{COMPLIANCE WITH ETHICAL STANDARDS}

The manuscript has been reviewed and approved by all co-authors. The authors certify that the paper has not been published (in part or in full) or submitted for publication elsewhere. We certify that each of the authors have made a substantial contribution so as to qualify to the authorship. All authors have disclosed all financial support for this work and other potential conflict of interests. This article does not contain any studies with human partecipants or animal performed by any of the authors.

\section{REFERENCES}

1. Magnussen RA, Lawrence JT, West RL, Toth AP, Taylor DC, Garrett WE. Graft size and patient age are predictors of early revision after anterior cruciate ligament reconstruction with hamstring autograft. Arthroscopy. 2012;28:526-31.

2. Maeda A, Shino K, Horibe S, Nakata K, Buccafusca G. Anterior cruciate ligament reconstruction with multistrand- 
ed autogenous semitendinosus tendon. Am J Sports Med. 1996;24:504-9.

3. Lavery KP, Rasmussen JF, Dhawan A: Five-strand hamstring autograft for anterior cruciate ligament reconstruction. Arthrosc Tech. 2014;3:e423-6.

4. Lee RJ, Ganley TJ: The 5-strand hamstring graft in anterior cruciate ligament reconstruction. Arthrosc Tech. 2014;3:e627-31.

5. Snow M, Cheung W, Mahmud J, Evans S, Holt C, Wang B, Chizari M. Mechanical assessment of two different methods of tripling hamstring tendons when using suspensory fixation. Knee Surg Sports Traumatol Arthrosc. 2012;20: 262-7.

6. Camarda L, Pitarresi G, Moscadini S, Marannano G, Sanfilippo A, D'Arienzo M. Effect of suturing the femoral portion of a four-strand graft during an ACL reconstruction. Knee Surg Sports Traumatol Arthrosc. 2014;22:1040-6.

7. Coleridge SD, Amis AA: A comparison of five tibial-fixation systems in hamstring-graft anterior cruciate ligament reconstruction. Knee Surg Sports Traumatol Arthrosc. 2004;12:391-7.

8. Weiss JA, Paulos LE. Mechanical testing of ligament fixation devices. Tech Orthop. 1999;14:14-21.

9. Jassem M, Rose AT, Meister K, Indelicato PA, Wheeler D. Biomechanical analysis of the effect of varying suture pitch in tendon graft fixation. Am J Sports Med. 2001;29: 734-7.

10. Krappinger D, Kralinger FS, El Attal R, Hackl W, Haid C. Modified Prusik knot versus whipstitch technique for soft tissue fixation in anterior cruciate ligament reconstruction: a biomechanical analysis. Knee Surg Sports Traumatol Arthrosc. 2007;15:418-23.

11. Honl M, Carrero V, Hille E, Schneider E, Morlock MM. Bone-patellar tendon-bone grafts for anterior cruciate ligament reconstruction: an in vitro comparison of mechanical behavior under failure tensile loading and cyclic submaximal tensile loading. Am J Sports Med. 2002;30:549-57.

12. Markolf KL, Gorek JF, Kabo JM, Shapiro MS. Direct measurement of resultant forces in the anterior cruciate ligament. An in vitro study performed with a new experimental technique. J Bone Joint Surg Am. 1990;72:557-67.

13. Padulo J, Oliva F, Frizziero A, Maffulli N. Muscles, Ligaments and Tendons Journal. Basic principles and recommendations in clinical and field science research: 2018 update. Muscles Ligaments Tendons J. 2018;8(3):305-307.

14. Camarda L, Grassedonio E, Albano D, Galia M, Midiri M, D'Arienzo M. MRI evaluation to predict tendon size for knee ligament reconstruction. Muscles Ligaments Tendons J. 2018;7(3):478-484.

15. Costa Astur D, Pires D, Parente T, Debieux P, Cohen Kaleka C, Skaf A, Cohen M. Short term evaluation of the hamstring graft diameter after ACL reconstruction. Muscles Ligaments Tendons J. 2019;9 (1):3-7

16. Hamner DL, Brown CH,Jr, Steiner ME, Hecker AT, Hayes WC. Hamstring tendon grafts for reconstruction of the anterior cruciate ligament: biomechanical evaluation of the use of multiple strands and tensioning techniques. J Bone Joint Surg Am. 1999;81: 549-57.

17. Jones PE, Schuett DJ: All-Inside Anterior Cruciate Ligament Reconstruction as a Salvage for Small or Attenuated Hamstring Grafts. Arthrosc Tech 2018;7:e453-7.

18. Vaillant ER, Parks BG, Camire LM, Hilton RY. FiveStrand versus Four-Strand Hamstring Tendon Graft Technique for Anterior Cruciate Ligament Reconstruction: A Biomechanical Comparison. J Knee Surg. 2017;30:916-9.

19. Broadhead ML, Singla AA, Bertollo N, Broe D, Walsh WR. A biomechanical comparison of 4-strand and 5-strand anterior cruciate ligament graft constructs. Orthop Rev (Pavia). 2017 Feb 20; 9(1): 6989.

20. Magen HE, Howell SM, Hull ML: Structural properties of six tibial fixation methods for anterior cruciate ligament soft tissue grafts. Am J Sports Med. 1999;27:35-43.

21. Donahue TL, Gregersen C, Hull ML, Howell SM. Comparison of viscoelastic, structural, and material properties of doublelooped anterior cruciate ligament grafts made from bovine digital extensor and human hamstring tendons. J Biomech Eng. 2001;123:162-9.

22. Brown GA, Pena F, Grontvedt T, Labadie D, Engebretsen L. Fixation strength of interference screw fixation in bovine, young human, and elderly human cadaver knees: influence of insertion torque, tunnel-bone block gap, and interference. Knee Surg Sports Traumatol Arthrosc. 1996;3:238-244. 